\title{
El estilo de socialización familiar como factor de prevención o riesgo para el consumo de sustancias y otros problemas de conducta en los adolescentes españoles
}

\section{The parenting style as protective or risk factor for substance use and other behavior problems among Spanish adolescents}

\author{
Isabel Martínez ${ }^{\star}$, María C. Fuentes ${ }^{* *}$, Fernando \\ GARCÍA $^{* *}$, IGNACIO MADRID*
}

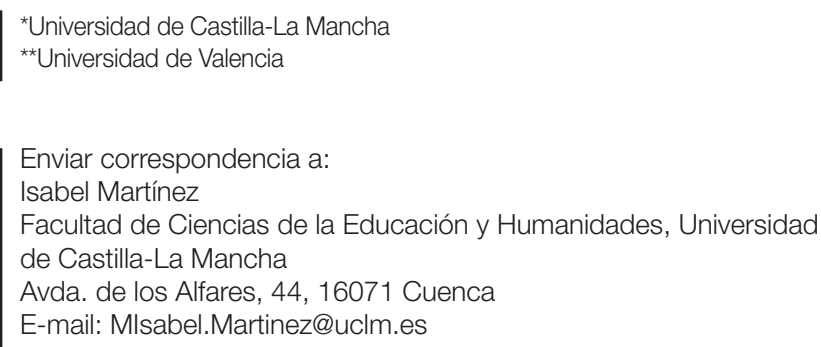

Enviar correspondencia a:

Isabel Martínez

Facultad de Ciencias de la Educación y Humanidades, Universidad de Castilla-La Mancha

Avda. de los Alfares, 44, 16071 Cuenca

E-mail: Mlsabel.Martinez@uclm.es

\section{Resumen}

El objetivo de este estudio fue analizar el estilo de socialización familiar como un factor de prevención o riesgo para el consumo de sustancias con una muestra de 673 adolescentes españoles de 14-17 años ( $M=15,49$; $D T=1,06)$ de los cuales el 51,7\% eran mujeres. Todos ellos completaron la Escala de Estilos de Socialización Parental en Adolescentes (ESPA29), y una escala de consumo de sustancias, además como criterios complementarios respondieron a una escala de comportamientos delictivos y a otra de conducta escolar disruptiva. Se aplicó un MANOVA factorial $(4 \times 2 \times 2)$ con el consumo de sustancias, los comportamientos delictivos y la conducta escolar disruptiva entre los estilos de socialización familiar, el sexo y la edad. Los resultados de los análisis identificaron al estilo de socialización parental indulgente como un factor de prevención del consumo de sustancias mientras que el estilo autoritario era identificado como un factor de riesgo. Además, los resultados referentes a los estilos parentales de prevención y riesgo para comportamientos delictivos y conducta escolar disruptiva siguen un patrón convergente con los del consumo de sustancias. Los resultados tienen implicaciones relevantes para el desarrollo de estrategias de prevención del consumo de sustancias centradas en el entorno familiar en adolescentes españoles y de entornos culturales similares, donde el estilo indulgente está mostrando actualmente tener un efecto beneficioso.

Palabras claves: consumo de sustancias, socialización, estilos parentales, factor de protección, factor de riesgo. recibido: Noviembre 2012 aceptado: Mayo 2013

\section{Abstract}

The aim of this study was to analyze the parental socialization styles as a protective or a risk factor for substance use in a sample of 673 Spanish adolescents (51.7\% were women) aged $14-17(M=15.49, S D=1.06)$. All participants completed the Parental Socialization Scale (ESPA29) and a scale of substance use. Additionally, they also completed a scale of delinquency and another one of school misconduct. A multivariate $(4 \times 2 \times 2)$ analysis of variance (MANOVA) was applied for substance use, delinquency and school misconduct with parenting style, sex and age. Results from this study showed that indulgent parenting style was a protective factor for substance use whereas authoritarian style was identified as a risk factor. Moreover, results from protective and risk parenting styles on delinquency and school misconduct were consistent with those obtained on substance use. These findings have important implications for the development of family-based substance use prevention programs among Spanish adolescents and other similar cultures where indulgent parenting style is currently displaying a beneficial impact.

Key words: substance use, socialization, parenting styles, protective factor, risk factor. 
E consumo de sustancias en la adolescencia se considera, actualmente, un serio problema de salud pública. Estudios recientes constatan la alta prevalencia que este problema alcanza entre los adolescentes de diferentes países, incluidos algunos situados en el área mediterránea de Europa (Calafat et al., 2011a; Calafat et al., 2011b). El consumo de sustancias en esta etapa tiene consecuencias más allá del deterioro a corto plazo de la salud física de los adolescentes. También conlleva riesgos a largo plazo para la salud física, psicológica y social del futuro adulto (Alati et al., 2010). La prevención de esta situación requiere conocer los diferentes aspectos que están influyendo, de modo que se puedan prevenir eficazmente los problemas derivados del consumo de sustancias que van desde aspectos relacionados con la salud física y psicológica hasta problemas sociales tan variados como el rendimiento laboral o la delincuencia juvenil y la conducta escolar disruptiva (Arce, Seijo, Fariña y Mohamed-Mohand, 2010; Calafat et al., 2011b; Lamborn, Mounts, Steinberg y Dornbusch, 1991; Thompson, Connelly, Thomas-Jones y Eggert, 2013).

Entre las variables que funcionan como factores de prevención o riesgo para el consumo de sustancias el entorno familiar ha sido considerado un importante factor (Grusec y Hastings, 2007; Darling y Cumsille, 2003). El papel de los padres, a través de estrategias educacionales y relacionales, ha sido destacado en distintas investigaciones recientes (Becoña, Martínez, Calafat, Juan, Fernández-Hermida y Secades-Villa, 2012; Cerdá, Rodríguez, Danet, Azarola, Toyos y Román, 2010; Pons y Buelga, 2011; Musitu, Jiménez y Murgui, 2007). Para clasificar las estrategias educacionales parentales se utilizan habitualmente los estilos de socialización, que se han definido tradicionalmente a partir de dos dimensiones independientes de la conducta paterna: exigencia y responsividad (Darling y Steinberg, 1993; Maccoby y Martin, 1983). La dimensión de exigencia se refiere a la extensión en que los padres utilizan el control, la supervisión y mantienen una posición de autoridad con sus hijos. Mientras que la dimensión de responsividad representa el grado en que los padres muestran afecto y aceptación a sus hijos, les dan apoyo y se comunican con ellos razonando (Martínez y García, 2007; Martínez, García y Yubero, 2007). Dependiendo del uso que los padres hacen de las prácticas educativas que caracterizan estas dos dimensiones se diferencian cuatro estilos de socialización parental: el estilo autoritario, que se caracteriza por una elevada utilización de prácticas de exigencia y bajo empleo de prácticas de responsividad; el estilo indulgente, caracterizado por un alto uso de prácticas de responsividad y bajo uso de prácticas de exigencia; el estilo autorizativo, que se define por una alta utilización de ambos tipos de prácticas; y el estilo negligente, que se caracteriza por un bajo empleo de ambos tipos de prácticas por los padres (Darling y Steinberg, 1993; Maccoby y Martin, 1983).

La investigación llevada a cabo fundamentalmente en los Estados Unidos con muestras que representan a la clase media blanca mayoritaria, ha mostrado reiteradamente que el estilo de socialización parental autorizativo es el óptimo en este contexto. Los hijos de padres autorizativos obtenían sistemáticamente las puntuaciones más altas en todos los criterios de ajuste y las más bajas en los criterios de desajuste (Darling y
Steinberg, 1993; Lamborn, et al., 1991). En el caso concreto del consumo de sustancias, los estilos autorizativo y autoritario actuaban como factores de prevención, mientras que los estilos negligente e indulgente suponían un factor de riesgo para que el hijo se involucrase en este comportamiento. Similares patrones se encontraron con las conductas disruptivas en la escuela, los comportamientos delictivos y otros problemas de ajuste del hijo al entorno social (Darling y Cumsille, 2003; Lamborn et al., 1991; Montgomery, Fisk y Craig, 2008; Steinberg, Lamborn, Darling, Mounts y Dornbusch, 1994). Aunque se ha comprobado que el grado de empleo de las prácticas parentales, que constituyen los diferentes estilos, varía en función tanto de la edad y el sexo del hijo como del sexo de los padres (Cerdá et al., 2010; Musitu y García, 2001), no se ha constatado que dichas variaciones modifiquen las relaciones entre los estilos parentales y distintos criterios de ajuste analizados en los hijos (Musitu y García, 2001, 2004; Lamborn et al., 1991). Sólo se ha constatado, de manera reiterada, una importante excepción que afecta la relación entre el estilo de socialización empleado y el ajuste del hijo, y que está siendo avalada actualmente por trabajos desarrollados en diferentes países: el contexto cultural donde se produce la socialización (Darling y Steinberg, 1993; Hindin, 2005; Kim y Rohner, 2003; Martínez et al., 2007; Martínez y García, 2008; Musitu y García, 2004).

Así, algunas investigaciones llevadas a cabo con minorías étnicas de Estados Unidos (Chao, 2001; Deater-Deckard, Dodge, Bates y Pettit, 1996) y con muestras de algunos países asiáticos (Dwairy, Achoui, Abouserfe y Farah, 2006; Quoss y Zhao, 1995) han obtenido resultados que apoyan el uso del estilo autoritario por parte de los padres. Además, recientemente, estudios llevados a cabo en algunos paises, entre los que se encuentra España, han encontrado que en determinados contextos culturales el estilo de socialización indulgente se asocia con los mismos o incluso mejores resultados en los hijos que el estilo autorizativo. De esta forma, se ha comprobado que no hay diferencias en el rendimiento académico de los adolescentes filipinos (Hindin, 2005) y de los coreo-americanos (Kim y Rohner, 2003) educados con estilos autorizativo e indulgente. El mismo patrón de resultados se ha encontrado para las competencias sociales de las adolescentes iranies (Kazemi, Ardabili y Solokian, 2010). Del mismo modo, en países de Sudamérica, como México (Villalobos, Cruz y Sánchez, 2004) y Brasil (Martínez y García, 2008), se ha encontrado que los hijos de padres indulgentes obtienen los mismos o mejores resultados que los hijos de padres autorizativos en distintas medidas de ajuste social y psicológico. Similares resultados han sido encontrados en varios países del sur de Europa, como son Turquía (Turkel y Tezer, 2008), Italia (Di Maggio y Zappulla, 2013), Portugal (Rodrigues, Veiga, Fuentes y García, 2013) y España (Martínez y Garcia, 2007; Musitu y García, 2004). En España los beneficios del estilo indulgente han sido probados sobre distintas variables psicológicas, como el autoconcepto (Musitu y García, 2004), los valores personales (Martínez y García, 2007) y el ajuste psicosocial (García y Gracia, 2009), y para prevenir algunas características, comportamientos y actitudes negativas, como los rasgos psicopáticos (López-Romero, Romero y Villar, 2012), la violencia hacia los padres (Gámez-Guadix, Jaureguizar, Almendros y Carrobles, 2012) o los prejuicios sexistas de los hijos (Garaigordobil y Aliri, 2012). 
Por tanto, la investigación reciente en España ha empezando a encontrar, de manera consistente, que un estilo de socialización indulgente, basado en el uso de aceptación y afecto y en la utilización del diálogo y el razonamiento con los hijos, tiende a relacionarse con mejor ajuste de los adolescentes que el uso de otros estilos de socialización parental (Alonso-Geta, 2012; de la Torre, Casanova, García, Carpio y Cerezo, 2011; García y Gracia, 2010). Estos trabajos, junto con los realizados en otras culturas diferentes a la anglosajona (Dor y Cohen-Fridel, 2010; Kazemi et al., 2010; Martínez y García, 2008; Turkel y Tezer, 2008), están cuestionando la generalización transcultural de los resultados de la investigación desarrollada en este área durante los últimos 50 años. El efecto de prevención o riesgo que los diferentes estilos de socialización parental puedan tener sobre el consumo de sustancias tiene particular interés, ya que en la actualidad se debate que algunas variables tradicionalmente consideradas de prevención para el consumo, como la alta autoestima, puedan estar actuando como tales (Fuentes, García, Gracia y Lila, 2011). De hecho, los pocos estudios españoles actuales que abordan la cuestión no permiten establecer claramente el efecto de prevención o de riesgo que los distintos estilos de socialización tienen sobre el consumo de sustancias y problemas de conducta relacionados, habiendo tanto estudios que apuntan al estilo autorizativo como factor de prevención (véase Cano, Solanas, Marí-Klose y Marí-Klose, 2012), como estudios que, por el contrario, señalan al estilo indulgente (véase García y Gracia, 2009).

En este trabajo, se analiza el efecto de prevención o de riesgo que los estilos de socialización parental tienen sobre el consumo de sustancias durante la adolescencia. Además se analizan otros dos criterios externos de ajuste del adolescente, las conductas delictivas y el comportamiento disruptivo escolar, que tradicionalmente siguen pautas de relación con los estilos de socialización similares al consumo de sustancias (Lamborn et al., 1991; Steinberg et al., 1994). Dado que los estudios recientes realizados en España (e.g., Martínez y Garcia, 2007; Musitu y García, 2004) y en contextos culturales similares (e.g., Martínez y García, 2008; Rodrigues, et al., 2013), han mostrado que, en general, el estilo indulgente es el que se asocia con mejor ajuste de los adolescentes, se espera que en el caso del consumo de sustancias y los problemas de conducta relacionados el patrón sea similar. Así, se espera que el estilo indulgente sea el estilo de socialización que actúe en mayor medida como factor de prevención del consumo de sustancias, igualando o superando al estilo autorizativo.

\section{Método}

\section{Participantes}

Para determinar el tamaño de la muestra se realizó un análisis de la potencia a priori asumiendo un tamaño del efecto bajomedio $(f=0,13)$, fijando los errores Tipo I y Tipo II en los límites convencionales, $a=0,05$ y $\beta=0,80$ (García, Pascual, Frías, Van Krunckelsven y Murgui, 2008) para las pruebas $F$ univariadas entre los cuatro estilos parentales. Se estimó que la muestra tendría que tener un tamaño mínimo de 652 participantes (Faul, Erdfelder, Buchner y Lang, 2009; García et al., 2008).
Los datos se recogieron en ocho centros educativos seleccionados por muestreo aleatorio simple de un listado completo de los centros de una comunidad autónoma española (i.e., Castilla la Mancha). Cuando los grupos (i.e., centros educativos) se seleccionan al azar, los elementos que componen los grupos (i.e., alumnos) serán similares a los que proporcionaría un sistema aleatorio (Kalton, 1983). Se contactó con los directores de los centros (ninguno negó su participación) y se consiguieron los habituales permisos paternos. El 97\% de los alumnos completaron los instrumentos, que se recogieron en sobres cerrados y anónimos por investigadores entrenados que supervisaron su adecuada cumplimentación. La muestra final fueron 673 participantes, 348 mujeres $(51,7 \%)$ y 325 hombres $(48,3 \%)$, con edades comprendidas entre 14 y 17 años $(M=$ 15,49; $D T=1,06)$.

\section{Instrumentos}

Consumo de sustancias y problemas de conducta relacionados. Para medir el consumo de sustancias en los adolescentes se utilizaron 4 items que valoran la cantidad de tabaco, alcohol, marihuana y otras sustancias (Chao, 2001; Lamborn et al., 1991) que el adolescente ha consumido en las últimas semanas (e.g., "En las últimas semanas ¿has consumido bebidas alcohólicas?") con una escala de respuesta que oscila entre 1 ("Nada") y 4 ("Mucho"). El coeficiente alfa para la escala fue 0,76 .

Además, también se midieron indicadores de la conducta escolar disruptiva y la conducta delictiva de los adolescentes (Chao, 2001; Lamborn et al., 1991). Para medir la conducta escolar disruptiva se utilizaron 5 ítems que indicaban la frecuencia de realización de gamberradas en el colegio (e.g., "Pegar a alguien o participar en peleas dentro del colegio/instituto") con una escala de respuesta de 1 ("Nunca") a 3 ("Dos 0 más veces"). La delincuencia se midió con 8 ítems que indicaban la implicación de los adolescentes en hechos pre-delictivos o delictivos (e.g., "Estropear coches de desconocidos") utilizando una escala de respuesta de 1 a 3 igual a la anterior. Los coeficientes alfa para las escalas de los dos índices analizados fueron: conducta escolar disruptiva, 0,68 y delincuencia, 0,80.

Escala de Estilos de Socialización Parental en Adolescentes ESPA29 (Musitu y García, 2001). Está compuesta por 212 ítems que evalúan los estilos de socialización familiar a través de la valoración que los adolescentes hacen de la actuación de sus padres en 29 situaciones representativas de la vida cotidiana familiar en la cultura occidental: 16 situaciones hacen referencia a las conductas de los hijos ajustadas a las normas familiares (e.g., "Si respeto los horarios establecidos en mi casa") y 13 se refieren conductas contrarias a dichas normas (e.g., "Si voy sucio y desastrado"). Para cada una de estas situaciones, los adolescentes valoran, con una escala de respuesta de 1 ("Nunca") a 4 ("Siempre"), la frecuencia con la que sus padres emplean las prácticas de afecto ("Me muestra cariño") e indiferencia ("Se muestra indiferente") ante sus comportamientos ajustados; y las prácticas de diálogo ("Habla conmigo"), displicencia ("Le da igual"), coerción verbal ("Me riñe"), coerción física ("Me pega") y privación ("Me priva de algo") ante sus comportamientos no adecuados a la norma. 
Con estas valoraciones se obtiene una medida global para cada padre en las dos dimensiones de la escala: Aceptación/Implicación y Severidad/Imposición, equivalentes a las dimensiones teóricas de responsividad y exigencia del modelo de socialización, y a partir de las cuales se tipifica el estilo de socialización parental como autorizativo, indulgente, autoritario y negligente. La puntuación en la dimensión aceptación/implicación se obtiene promediando las subescalas de afecto, dialogo, indiferencia y displicencia (en las dos últimas se invierte la puntuación porque están inversamente relacionadas con la dimensión). La puntuación en la dimensión severidad/imposición se obtiene promediando las subescalas de coerción verbal, coerción física y privación.

La estructura factorial del ESPA29 se ha confirmado en diferentes estudios (Martínez, Musitu, García y Camino, 2003; Martínez, García, Musitu y Yubero, 2012), así como la ortogonalidad de su dimensiones (Martínez, Garcia, Camino y Camino, 2011; Musitu y García, 2001). El coeficiente alfa de las dos dimensiones principales fue: aceptación/implicación, 0,96 ; y severidad/imposición, 0,96 y el de las siete subescalas fue: afecto, 0,95; indiferencia, 0,93; diálogo, 0,93; displicencia, 0,90 ; coerción verbal, 0,93; coerción física, 0,95; y privación, 0,95 .

\section{Resultados}

\section{Clasificación en los estilos de socialización familiar}

Se tipificó el estilo de socialización parental (autorizativo, indulgente, autoritario o negligente) por el sistema habitual de dicotomizar a partir de la mediana (Chao, 2001) las puntuaciones promediadas del padre y la madre (Lamborn et al., 1991) en las dos dimensiones de la escala, aceptación/implicación y severidad/imposición controlando el sexo y la edad del hijo (Musitu y García, 2001). De esta forma, las familias autorizativas son las que puntúan por encima del percentil 50 en ambas dimensiones, las familias indulgentes puntúan por encima del percentil 50 en aceptación/implicación y por debajo de éste en severidad/imposición, las familias autoritarias puntúan por debajo del percentil 50 en aceptación/implicación y por encima de ese percentil en severidad/imposición, y las familias negligentes puntúan por debajo del percentil 50 en ambas dimensiones. En la Tabla 1 se ha presentado la distribución de las familias en los cuatro estilos parentales. La distribución por estilos parentales fue estadisticamente homogénea, $\chi^{2}(3)=$ $2,33, p>0,05$, como también lo fue la distribución cruzada con el sexo, $\chi^{2}(3)=0,71, p>0,05$, y con la edad del hijo, $\chi^{2}(9)=$ $3,50, p>0,05$.

\section{Estilos parentales y consumo de sustancias}

Para analizar las relaciones entre los estilos de socialización parental y el consumo de sustancias, se aplicó un diseño factorial multivariado $(4 \times 2 \times 2)$ con el consumo de sustancias, la conducta escolar disruptiva y el indicador de delincuencia como variables dependientes y los estilos de socialización
Tabla 1

Distribución por frecuencias de los estilos de socialización familiar, medias y desviaciones típicas en los dos ejes: Aceptación/Implicación y Severidad/Imposición

\begin{tabular}{llllll}
\hline & Total & Indulgente & Autorizativo & Autoritario & Negligente \\
\hline Frecuencia & 673 & 168 & 184 & 157 & 164 \\
Porcentaje & 100 & 25,0 & 27,3 & 23,3 & 24,4 \\
Aceptación/ & & & & & \\
Implicación & & & & & \\
Media & 3,07 & 3,37 & 3,41 & 2,74 & 2,72 \\
DT & 0,43 & 0,24 & 0,26 & 0,29 & 0,29 \\
Severidad/ & & & & & \\
Imposición & & & & & \\
Media & 1,70 & 1,41 & 1,95 & 2,00 & 1,41 \\
DT & 0,37 & 0,19 & 0,26 & 0,32 & 0,17 \\
\hline
\end{tabular}

Nota. Las puntuaciones en aceptación/implicación y severidad/imposición oscilan de 1 a 4 .

Tabla 2

MANOVA Factorial $\left(4^{a} \times 2^{b} \times 2^{c}\right)$ para problemas de conducta consumo de sustancias, conducta escolar disruptiva y delincuencia

\begin{tabular}{|c|c|c|}
\hline Fuente de variación & $\wedge$ & $F$ \\
\hline (A) Estilo parental a & 0,963 & $F(9,1582,08)=2,74^{* *}$ \\
\hline (B) Sexo ${ }^{b}$ & 0,919 & $\nexists(3,650,00)=19,11^{* * *}$ \\
\hline (C) Edad ${ }^{c}$ & 0,987 & $A(3,650,00)=2,79^{*}$ \\
\hline$A \times B$ & 0,986 & $\not(9,1582,08)=1,06$ \\
\hline$A \times C$ & 0,986 & $\not(9,1582,08)=1,05$ \\
\hline$B \times C$ & 0,991 & $F(3,650,000)=2,06$ \\
\hline$A \times B \times C$ & 0,990 & $H(9,1582,08)=0,76$ \\
\hline
\end{tabular}

${ }^{a} a_{1}$, indulgente, $a_{2^{\prime}}$ autorizativo, $a_{3^{\prime}}$ autoritario, $a_{4^{\prime}}$ negligente.

${ }^{b} b_{1}$, hombres, $b_{2}$, mujeres. ${ }^{c} c_{1}, 14-15$ años, $c_{2}, 16-17$ años.

${ }^{*} p<0,05,{ }^{* *} p<0,01,{ }^{* * *} p<0,001$

parental (autorizativo, indulgente, autoritario y negligente), el sexo (hombre vs. mujer) y la edad (14 - 15 años vs. $16-17$ años) como variables independientes. Se aplicaron los posteriores ANOVAs y las pruebas post-hoc de Bonferroni para analizar las diferencias entre medias, corrigiendo así la tasa del error de Tipo I.

En los resultados del MANOVA (véase Tabla 2) se encontraron diferencias estadisticamente significativas de los efectos principales del estilo parental de socialización, la edad y el sexo. No se encontró ningún efecto de interacción estadísticamente significativo $(a=0,05)$.

Efectos principales de las variables demográficas. Los resultados revelan que la variable edad produce diferencias significativas sobre el consumo de sustancias, mostrando que los adolescentes entre 16 y 17 años obtienen puntuaciones más altas que los adolescentes entre 14 y 15 años (véase Tabla 3), con un tamaño del efecto bajo (Faul, et al., 2009). Además, la conducta escolar disruptiva y la delincuencia mostraron diferencias estadísticamente significativas en función del sexo, con tamaños del efecto medios (véase Tabla 3), correspondiendo a los hombres las puntuaciones más altas en ambos índices. 
Tabla 3

Medias, desviaciones típicas (entre paréntesis) y valores F entre estilos de socialización, sexo y edad en consumo de sustancias, conducta escolar disruptiva y delincuencia

\begin{tabular}{|c|c|c|c|c|c|c|c|c|c|c|c|}
\hline & \multicolumn{5}{|c|}{ Estilo Parental \# } & \multicolumn{3}{|l|}{ Sexo } & \multicolumn{3}{|l|}{ Edad } \\
\hline & Indulgente & Autorizativo & Autoritario & Negligente & $f(3,664)[f]$ & Hombre & Mujer & f(1, 666) $[f]$ & 14-15 años & 16-17 años & $f(1,666)[f]$ \\
\hline \multirow[t]{2}{*}{ Consumo de sustancias } & $1,37^{2}$ & 1,44 & $1,61^{1}$ & 1,54 & $4,73^{* *}$ & 1,50 & 1,48 & 0,10 & 1,43 & 1,55 & $6,44^{*}$ \\
\hline & $(0,54)$ & $(0,56)$ & $(0,70)$ & $(0,66)$ & {$[0,14]$} & $(0,67)$ & $(0,57)$ & {$[0,01]$} & $(0,57)$ & $(0,66)$ & {$[0,10]$} \\
\hline Conducta escolar & $1,35^{2}$ & $1,36^{2}$ & $1,49^{1}$ & 1,45 & $3,82^{*}$ & 1,52 & 1,31 & $41,49^{* * *}$ & 1,40 & 1,42 & 0,32 \\
\hline disruptiva & $(0,45)$ & $(0,40)$ & $(0,45)$ & $(0,46)$ & {$[0,13]$} & $(0,48)$ & $(0,37)$ & {$[0,24]$} & $(0,42)$ & $(0,46)$ & {$[0,02]$} \\
\hline Delincuencia & $(0,39)$ & $(0,33)$ & $(0,42)$ & $(0,42)$ & {$[0.16]$} & $(0,43)$ & $(0,33)$ & {$[0,20]$} & $(0,38)$ & $(0,41)$ & {$[0,05]$} \\
\hline
\end{tabular}

* Prueba de Bonferroni, $\mathrm{a}=0,05,1>2$.

${ }^{*} p<0,05,{ }^{* *} p<0,01,{ }^{* * *} p<0,001$

Efectos principales de los estilos parentales. En el consumo de sustancias los hijos de padres indulgentes obtuvieron las puntuaciones más bajas en comparación con los hijos de familias autoritarias $(a=0,05)$, los tamaños del efecto detectados fueron entre bajos y medios, de 0,13 y 0,16 (véase Tabla 3). Además, también los otros dos indicadores evaluados mostraron diferencias estadisticamente significativas en función del estilo de socialización parental. En el caso de la conducta escolar disruptiva y los comportamientos delictivos, los adolescentes que definieron a sus padres como indulgentes y autorizativos obtuvieron las puntuaciones más bajas en comparación con los adolescentes de familias autoritarias $(a=0,05)$.

\section{Discusión}

Los resultados constatan que el estilo de socialización utilizado por los padres está relacionado con el consumo de sustancias. La dirección de esta relación confirma que el estilo de socialización indulgente actúa como un factor de prevención para el consumo de sustancias en adolescentes españoles, los hijos de hogares indulgentes manifiestan ser los que menos sustancias, como el alcohol, tabaco o marihuana, consumen frente a los educados en hogares autoritarios, que serian los más propensos a consumir este tipo de sustancias. Además, aunque en esta investigación los adolescentes educados con los estilos autorizativo y negligente no se diferencian significativamente en el consumo de sustancias con los educados con ningún otro estilo, se observa que el consumo de los adolescentes educados con un estilo autorizativo se encuentra próximo a los educados con un estilo indulgente, y el consumo de los educados de manera negligente está próximo a los educados con un estilo autoritario. Hay que destacar que los resultados de este estudio contrastan notoriamente con los encontrados en muestras de adolescentes caucásicos en los Estados Unidos, donde, manifiestamente, el estilo parental indulgente (junto con el negligente) constituía un factor de riesgo y el estilo parental autoritario (junto con el autorizativo) era un factor de protección para el consumo de sustancias (Darling y Cumsille, 2003; Lamborn et al., 1991; Montgomery et al., 2008; Steinberg et al., 1994). Además, de manera congruente con los resultados sobre el consumo de sustancias, en el caso de la conducta escolar disruptiva y el comportamiento delictivo, los adolescentes educados en hoga- res indulgentes y autorizativos son menos propensos a involucrarse en estos comportamientos que los educados en familias autoritarias, que manifiestan ser los que más se involucran, con lo cual los estilos indulgente y autorizativo serian los que, en mayor medida, actuarían como factores de prevención de estos problemas de conducta.

Por tanto, los resultados sugieren que el estilo de socialización indulgente resultaría el más recomendable por asociarse con menos problemas de conducta en indicadores de salud tan destacables como son el bajo consumo de sustancias y otros comportamientos asociados como los bajos índices de conducta escolar disruptiva y el comportamiento delictivo (Lamborn et al., 1991). De esta manera, se extienden a la prevención de estos problemas de conducta los resultados de estudios recientes que apoyan la utilización del estilo indulgente en el contexto español en aspectos tan variados como el autoconcepto (Musitu y García, 2004), los valores personales (Martínez y García, 2007) el ajuste psicosocial (Garcia y Gracia, 2009), los rasgos psicopáticos (López-Romero et al., 2012), la violencia hacia los padres (Gámez-Guadix et al., 2012) o los prejuicios sexistas de los hijos (Garaigordobil y Aliri, 2012).

Los datos obtenidos subrayan el beneficio que implica el componente de afecto y comunicación empática (Jiménez, Murgui, Estévez y Musitu, 2007) con los hijos, aspectos que comparten los estilos indulgente y autorizativo. Parece ser que si bien el estilo parental autorizativo que, aunque comparte con el autoritario el componente impositivo, hace uso del razonamiento y el afecto, supone una mejora frente este estilo autoritario, constatada en el presente estudio en los problemas conductuales de delincuencia y conducta escolar disruptiva, también es cierto que, en el contexto español, este componente de actuación parental impositivo no constituye una mejora respecto al estilo indulgente, que únicamente emplea el componente de afecto, empatía y diálogo para controlar satisfactoriamente la conducta de sus hijos. De hecho, en el caso del consumo de sustancias la conducta impositiva no se relaciona con ningún aspecto beneficioso (Lamborn et al., 1991), ya que en este caso el estilo indulgente, únicamente a través del empleo del afecto y el dialogo, es el que mejor parece actuar como factor de prevención. Por lo tanto, las acciones impositivas parentales no supone, ninguna ventaja sobre el control basado en el razonamiento, que repre- 
senta la relación de los padres que argumentan con su hijo con el fin de que interiorice los comportamientos adecuados.

Finalmente, los datos obtenidos también coinciden con la mayoría de los estudios realizados en este área de investigación (e.g., Lamborn et al., 1991; Martínez et al., 2007) en el hecho de que la relación entre estilos de socialización y criterios de ajuste no cambia en función de variaciones en las variables demográficas, no mostrando esta relación interacción, en nuestro estudio, con el sexo y la edad del hijo, pese a que sí se han constatado variaciones en las variables criterio (consumo de sustancias y otros problemas de conducta) en función de las dos variables demográficas consideradas, congruentes con las encontradas inicialmente por Lamborn et al. (1991) que mostraban la mayor presencia de problemas de conducta en los chicos frente a las chicas. Asimismo, el tamaño del efecto detectado en este estudio es similar al de otros estudios clásicos realizados en Estados Unidos (Lamborn et al., 1991) y España (Martínez y García, 2008).

Con las habituales limitaciones de este tipo de investigación, ya que se trata de un estudio con metodología no-experimental (Pérez, Navarro y Llobell, 1999; Veiga, García, Neto y Almeida, 2009) los resultados de este trabajo refuerzan la idea de que en el contexto español la socialización parental requiere de importantes esfuerzos en aspectos muchas veces relegados a un segundo plano, como la comunicación con los hijos, las relaciones cordiales, el interés por sus problemas y la explicación razonada de las consecuencias de sus actos para que puedan adquirir la condición de personas autónomas y responsables capaces de interiorizar los valores y las normas sociales (Martínez et al., 2007; Martínez y García, 2007, 2008). Finalmente, las actuaciones impositivas parentales aunque persigan como fin aspectos beneficiosos para la salud y bienestar de los hijos, como evitar problemas conductuales tan serios como el consumo de drogas, lejos de ser imprescindibles (Lamborn et al., 1991) estas actuaciones parentales parecen contribuir poco en la cultura española (García y Gracia, 2009; Martínez y García, 2008; Musitu y García, 2004). Estos aspectos deberían considerarse cuando se diseñen las estrategias de prevención (Galdós y Sánchez, 2010; Saiz, Álvaro y Martínez, 2011) para tratar problemas de conducta, como el consumo de sustancias, en adolescentes en España y en entornos culturales similares (Burkhart, 2011; Faggiano, 2011), teniendo en cuenta que no es posible extrapolar, sin más, esquemas de socialización parental idóneos en otros contextos.

\section{Reconocimientos}

Este artículo se ha realizado en el marco del Programa VALi+d para investigadores en formación del Plan General Estratégico de Ciencia y Tecnología de la Comunidad Valenciana (2009-2013) (ACIF/2010/282).

\section{Conflicto de intereses}

Los autores declaran que no existe ningún conflicto de interés.

\section{Referencias}

Alati, R., Maloney, E., Hutchinson, D. M., Najman, J. M., Mattick, R. P., Bor, W. y Williams, G. M. (2010). Do maternal parenting practices predict problematic patterns of adolescent alcohol consumption? Addiction, 105, 872-880. doi:10.1111/j.1360-0443.2009.02891.x

Alonso-Geta, P. M. P. (2012). Parenting style in Spanish parents with children aged 6 to 14 [La socialización parental en padres españoles con hijos de 6 a 14 años]. Psicothema, 24, 371-376.

Arce, R., Seijo, D., Fariña, F. y Mohamed-Mohand, L. (2010). Comportamiento antisocial en menores: Riesgo social y trayectoria natural de desarrollo [Antisocial behavior in adolescents: Social risk and natural developmental trajectory]. Revista Mexicana de Psicología, 27, 127-142.

Becoña, E., Martínez, U., Calafat, A., Juan, M., Fernández-Hermida, J. R. y Secades-Villa, R. (2012). Parental styles and drug use: A review. Drugs: Education, Prevention and Policy, 19, 1-10. doi:10.31 09/09687637.2011.631060

Burkhart, G. (2011). Environmental drug prevention in the EU. Why is it so unpopular? [Prevención ambiental de drogas en la Unión Europea ¿Por qué es tan impopular este tipo de prevención?]. Adicciones, 23, 87-100.

Calafat, A., Blay, N. T., Hughes, K., Bellis, M., Juan, M., Duch, M. y Kokkevi, A. (2011a). Nightlife young risk behaviours in Mediterranean versus other European cities: are stereotypes true? European Journal of Public Health, 21, 311-315. doi:10.1093/eurpub/ckq141

Calafat, A., Mantecón, A., Juan, M., Adrover-Roig, D., Blay, N. y Rosal, F. (2011b). Violent behaviour, drunkenness, drug use, and social capital in nightlife contexts [Comportamiento violento, embriaguez, uso de drogas y capital social en los contextos de ocio nocturno]. Psychosocial Intervention, 20, 45-51. doi:10.5093/ in2011v20n1a4

Cano, A. J., Solanas, S. E., Marí-Klose, M. y Marí-Klose, P. (2012). Psychosocial risk factors in adolescent tobacco use: Negative mood-states, peer group and parenting styles [Factores de riesgo psicosociales en el consumo de tabaco de los adolescentes: Estados de ánimo negativos, grupo de iguales y estilos parentales]. Adicciones, 24, 309-317.

Cerdá, J. C. M., Rodriguez, M. A. P., Danet, A., Azarola, A. R., Toyos, N. G. y Román, P. R. (2010). Posicionamiento de padres y madres ante el consumo de alcohol en población de 12 a 17 años en el ámbito urbano de seis Comunidades Autónomas [Parent's positioning towards alcohol consumption in 12 to 17 years old adolescents from six urban areas in Spain]. Gaceta Sanitaria, 24, 53-58. doi:10.1016/j.gaceta.2009.09.016

Chao, R. K. (2001). Extending research on the consequences of parenting style for Chinese Americans and European Americans. Child Development, 72, 1832-1843. doi:10.1111/1467-8624.00381

Darling, N. y Cumsille, P. (2003). Theory, measurement, and methods in the study of family influences on adolescent smoking. Addiction, 98, 21-36.

Darling, N. y Steinberg, L. (1993). Parenting style as context: An integrative model. Psychological Bulletin, 113, 487-496. doi:10.1037/0033-2909.113.3.487

de la Torre, M. J., Casanova, P. F., García, M. C., Carpio, M. V. y Cerezo, M. T. (2011). Parenting styles and stress in students of compulsory 
secondary education [Estilos educativos paternos y estrés en estudiantes de educación secundaria obligatoria]. Behavioral Psychology, 19, 577-590.

Deater-Deckard, K., Dodge, K. A., Bates, J. E. y Pettit, G. S. (1996). Physical discipline among African American and European American mothers: Links to children's externalizing behaviors. Developmental Psychology, 32, 1065-1072. doi:10.1037/0012-1649.32.6.1065

Di Maggio, R. y Zappulla, C. (2013). Mothering, fathering, and Italian adolescents' problem behaviors and life satisfaction: Dimensional and typological approach. Journal of Child and Family Studies. doi:10.1007/s10826-013-9721-6

Dor, A. y Cohen-Fridel, S. (2010). Preferred parenting styles: Do Jewish and Arab-Israeli emerging adults differ? Journal of Adult Development, 17, 146-155. doi:10.1007/s10804-010-9092-9

Dwairy, M., Achoui, M., Abouserfe, R. y Farah, A. (2006). Parenting styles, individuation, and mental health of Arab adolescents: A third crossregional research study. Journal of Cross-Cultural Psychology, 37, 262-272. doi:10.1177/0022022106286924

Faggiano, F. (2011). Reaction to the article: Environmental drug prevention in the EU - why is it so unpopular? by Gregor Burkhart [Reacción a "Prevención ambiental de drogas en la Unión Europea. ¿Por qué es tan impopular este tipo de prevención? de Gregor Burkhart»]. Adicciones, 23, 101-102.

Faul, F., Erdfelder, E., Buchner, A. y Lang, A. G. (2009). Statistical power analyses using $\mathrm{G}^{*}$ Power 3.1: Tests for correlation and regression analysess. Behavior Research Methods, 41, 1149-1160. doi:10.3758/ BRM.41.4.1149

Fuentes, M. C., García, F., Gracia, E. y Lila, M. (2011). Autoconcepto y consumo de sustancias en la adolescencia [Self-concept and drug use in adolescence]. Adicciones, 23, 237-248.

Galdós, J. S. y Sánchez, I. M. (2010). Relationship between cocaine dependence treatment and personal values of openness to change and conservation [Relación del tratamiento por dependencia de la cocaina con los valores personales de apertura al cambio y conservación]. Adicciones, 22, 51-58.

Gámez-Guadix, M., Jaureguizar, J., Almendros, C. y Carrobles, J. A. (2012). Parenting styles and child to parent violence in Spanish population [Estilos de socialización familiar y violencia de hijos a padres en población española]. Behavioral Psychology, 20, 585-602.

Garaigordobil, M. y Aliri, J. (2012). Parental socialization styles, parents' educational level, and sexist attitudes in adolescence. Spanish Journal of Psychology, 15, 592-603. doi:10.5209/rev_SJOP.2012.v15. n2.38870

Garcia, F. y Gracia, E. (2009). Is always authoritative the optimum parenting style? Evidence from Spanish families. Adolescence, 44(173), 101-131.

Garcia, F. y Gracia, E. (2010) ¿Qué estilo de socialización parental es el idóneo en España? Un estudio con niños y adolescentes de 10 a 14 años [What is the optimum parental socialisation style in Spain? A study with children and adolescents aged 10-14 years]. Infancia y Aprendizaje, 33, 365-384. doi:10.1174/021037010792215118
Garcia, J. F., Pascual, J., Frias, M. D., Van Krunckelsven, D. y Murgui, S. (2008). Design and power analysis: $n$ and confidence intervals of means. Psicothema, 20, 933-938.

Grusec, J. E. y Hastings, P. D. (2007). Handbook of socialization: Theory and research. New York: Guilford Press.

Hindin, M. J. (2005). Family dynamics, gender differences and educational attainment in Filipino adolescents. Journal of Adolescence, 28, 299-316. doi:10.1016/j.adoleseence.2004.12.003

Jiménez, T. I., Murgui, S., Estévez, E. y Musitu, G. (2007). Comunicación familiar y comportamientos delictivos en adolescentes españoles: El doble rol mediador de la autoestima [Family communication and delinquent behavior among Spanish adolescents: The mediating role of self-esteem]. Revista Latinoamericana de Psicología, 39, 473-485.

Kalton, G. (1983). Introduction to survey sampling. Beverly Hills, CA: Sage.

Kazemi, A., Ardabili, H. E. y Solokian, S. (2010). The association between social competence in adolescents and mothers' parenting style: A cross sectional study on Iranian girls. Child and Adolescent Social Work Journal, 27, 395-403. doi:10.1007/s10560-010-0213-x

Kim, S. I. y Rohner, R. P. (2003). Perceived parental acceptance and emotional empathy among university students in Korea. Journal of Cross-Cultural Psychology, 34, 723-735. doi:10.1177/0022022102033002001

Lamborn, S. D., Mounts, N. S., Steinberg, L. y Dornbusch, S. M. (1991). Patterns of competence and adjustment among adolescents from authoritative, authoritarian, indulgent, and neglectful families. Child Development, 62, 1049-1065. doi:10.1111/j.1467-8624.1991. tb01588.x

López-Romero, L., Romero, E. y Villar, P. (2012). Relationships between parenting styles and psychopathic traits in childhood [Relaciones entre estilos educativos parentales y rasgos psicopáticos en la infancia]. Behavioral Psychology, 20, 603-623.

Maccoby, E. E. y Martin, J. A. (1983). Socialization in the context of the family: Parent-child interaction. In P. H. Mussen (Ed.), Handbook of child psychology (pp. 1-101). New York: Wiley.

Martínez, I., Garcia, J. F., Camino, L. y Camino, C. (2011). Socialização parental: Adaptação ao Brasil da escala ESPA29 [Parental socialization: Brazilian adaptation of the ESPA29 scale]. Psicologia: Reflexão e Crítica, 24, 640-647. doi:10.1590/S010279722011000400003

Martínez, I., García, F., Musitu, G. y Yubero, S. (2012). Family socialization practices: Factor confirmation of the Portuguese version of a scale for their measurement. Revista de Psicodidactica, 17, 159-178. doi:10.1387/RevPsicodidact.1306

Martinez, I. y Garcia, J. F. (2007). Impact of parenting styles on adolescents' self-esteem and internalization of values in Spain. Spanish Journal of Psychology, 10, 338-348.

Martinez, I. y García, J. F. (2008). Internalization of values and selfesteem among Brazilian teenagers from authoritative, indulgent, authoritarian, and neglectful homes. Adolescence, 43(169), 13-29.

Martínez, I., García, J. F. y Yubero, S. (2007). Parenting styles and adolescents' self-esteem in Brazil. Psychological Reports, 100, 731745. doi:10.2466/pr0.100.3.731-745 
Martínez, I., Musitu, G., García, J. F. y Camino, L. (2003). Un análisis intercultural de los efectos de la socialización familiar en el autoconcepto: España y Brasil [A cross-cultural analysis of the effects of family socialization on self-concept: Spain and Brazill. Psicologia, Educação e Cultura, 7, 239-259.

Montgomery, C., Fisk, J. E. y Craig, L. (2008). The effects of perceived parenting style on the propensity for illicit drug use: The importance of parental warmth and control. Drug and Alcohol Review, 27, 640649. doi:10.1080/09595230802392790

Musitu, G. y Garcia, F. (2001). ESPA29: Escala de estilos de socialización parental en la adolescencia [ESPA29: Parental socialization scale in adolescence]. Madrid, Spain: Tea.

Musitu, G. y García, J. F. (2004). Consecuencias de la socialización familiar en la cultura española [Consequences of the family socialization in the Spanish culture]. Psicothema, 16, 288-293.

Musitu, G., Jiménez, T. I. y Murgui, S. (2007). El rol del funcionamiento familiar y del apoyo social en el consumo de sustancias de los adolescentes [The role of family functioning and social support in adolescent's substance consumption]. Revista Española de Drogodependencias, 32, 370-380.

Pérez, J. F. G., Navarro, D. F. y Llobell, J. P. (1999). Potencia estadistica del diseño de Solomon [Statistical power of Solomon design]. Psicothema, 11, 431-436.

Pons, J. y Buelga, S. (2011). Factores asociados al consumo juvenil de alcohol: Una revisión desde una perspectiva psicosocial y ecológica [Factors associated with youth alcohol consumption: A review from a psychosocial and ecological perspective]. Psychosocial Intervention, 20, 75-94. doi:10.5093/in2011v20n1a8

Quoss, B. y Zhao, W. (1995). Parenting styles and children's satisfaction with parenting in China and the United States. Journal of Comparative Family Studies, 26, 265-280.

Rodrigues, Y., Veiga, F., Fuentes, M.C., y García, F. (2013). Parenting and adolescents' self-esteem: The Portuguese context. Revista de Psicodidáctica, 18, 395-416. doi:10.1387/RevPsicodidact.6842

Saiz J., Álvaro J. L. y Martínez I. (2011). Relation between personality traits and personal values in cocaine's dependent patients. Adicciones, 23, 125-132.

Steinberg, L., Lamborn, S. D., Darling, N., Mounts, N. S y Dornbusch, S. M. (1994). Over-Time changes in adjustment and competence among adolescents from authoritative, authoritarian, indulgent, and neglectful families. Child Development, 65, 754-770. doi:10.1111/j.1467-8624.1994.tb00781.x

Thompson, E. A., Connelly, C. D., Thomas-Jones, D. y Eggert, L. L. (2013). School difficulties and co-occurring health risk factors: Substance use, aggression, depression, and suicidal behaviors.Journal of Child and Adolescent Psychiatric Nursing, 26, 74-84. doi:10.1111/jcap.12026

Turkel, Y. D. y Tezer, E. (2008). Parenting styles and learned resourcefulness of Turkish adolescents. Adolescence, 43(169), 143-152.

Veiga, F. H., Garcia, F., Neto, F. y Almeida, L. (2009). The differentiation and promotion of students' rights in Portugal. School Psychology International, 30, 421-436. doi:10.1177/0143034309106947

Villalobos, J. A., Cruz, A. V. y Sánchez, P. R. (2004). Estilos parentales y desarrollo psicosocial en estudiantes de Bachillerato [Parental styles and psychosocial development in High-School students]. Revista Mexicana de Psicología, 21, 119-129. 